\author{
Marquette University \\ e-Publications@Marquette
}

$10-31-2018$

\title{
Several Levels of Theory for Description of Isotope Effects in Ozone: Effect of Resonance Lifetimes and Channel Couplings
}

Alexander Teplukhin

Marquette University

Dmitri Babikov

Marquette University, dmitri.babikov@marquette.edu

Igor Gayday

Marquette University

Follow this and additional works at: https://epublications.marquette.edu/chem_fac

Part of the Chemistry Commons

\section{Recommended Citation}

Teplukhin, Alexander; Babikov, Dmitri; and Gayday, Igor, "Several Levels of Theory for Description of Isotope Effects in Ozone: Effect of Resonance Lifetimes and Channel Couplings" (2018). Chemistry Faculty Research and Publications. 958.

https://epublications.marquette.edu/chem_fac/958 


\section{Several levels of theory for description of isotope effects in ozone: Effect of resonance lifetimes and channel couplings}

Cite as: J. Chem. Phys. 149, 164302 (2018); https://doi.org/10.1063/1.5042590

Submitted: 02 June 2018 . Accepted: 25 September 2018 . Published Online: 22 October 2018

Alexander Teplukhin (D), Igor Gayday, and Dmitri Babikov
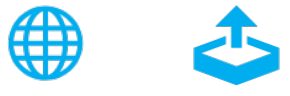

\section{ARTICLES YOU MAY BE INTERESTED IN}

Dipole-bound excited states and resonant photoelectron imaging of phenoxide and thiophenoxide anions

The Journal of Chemical Physics 149, 164301 (2018); https://doi.org/10.1063/1.5049715

Quantum mechanical study of the ${ }^{16} \mathrm{O}+{ }^{18} \mathrm{O}{ }^{18} \mathrm{O} \rightarrow{ }^{16} \mathrm{O}{ }^{18} \mathrm{O}+{ }^{18} \mathrm{O}$ exchange reaction: Integral cross sections and rate constants

The Journal of Chemical Physics 149, 214304 (2018); https://doi.org/10.1063/1.5053469

Erratum: "Several levels of theory for description of isotope effects in ozone: Effect of resonance lifetimes and channel couplings" []. Chem. Phys. 149, 164302 (2018)]

The Journal of Chemical Physics 149, 209901 (2018); https://doi.org/10.1063/1.5079540 


\title{
Several levels of theory for description of isotope effects in ozone: Effect of resonance lifetimes and channel couplings
}

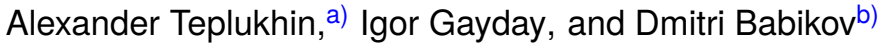 \\ Department of Chemistry, Marquette University, Wehr Chemistry Building, Milwaukee, \\ Wisconsin 53201-1881, USA
}

(Received 2 June 2018; accepted 25 September 2018; published online 22 October 2018)

\begin{abstract}
In this paper, two levels of theory are developed to determine the role of scattering resonances in the process of ozone formation. At the lower theory level, we compute resonance lifetimes in the simplest possible way, by neglecting all couplings between the diabatic vibrational channels in the problem. This permits to determine the effect of "shape" resonances, trapped behind the centrifugal barrier and populated by quantum tunneling. At the next level of theory, we include couplings between the vibrational channels, which permits to determine the role of Feshbach resonances and interaction of different reaction pathways on the global PES of ozone. Pure shape resonances are found to contribute little to the overall recombination process since they occur rather infrequently in the spectrum, in the vicinity of the top of the centrifugal barrier only. Moreover, the associated isotope effects are found to disagree with experimental data. By contrast, Feshbach-type resonances are found to make dominant contribution to the process. They occur in a broader range of spectrum, and their density of states is much higher. The properties of Feshbach resonances are studied in detail. They explain the isotopic $\zeta$-effect, giving theoretical prediction in good agreement with experiments for both singly and doubly substituted ozone molecules. Importantly, Feshbach resonances also contribute to the isotopic $\eta$-effect, moving theoretical predictions in the right direction. Some differences with experimental data remain, which indicates that there may be another additional source of the $\eta$-effect. Published by AIP Publishing. https://doi.org/10.1063/1.5042590
\end{abstract}

\section{INTRODUCTION}

The recombination reaction that forms ozone exhibits anomalously large variations of rate coefficient, due to isotopic substitutions, which can be characterized by introducing two parameters for two distinct isotope effects: $\zeta$-effect and $\eta$-effect. Namely, for the singly substituted isotopomer of ozone,

$$
\begin{aligned}
\zeta & =\frac{k^{A}}{K_{\mathrm{ex}} k^{B}}, \\
\eta & =\frac{k^{A}+K_{\mathrm{ex}} k^{B}}{2 k^{S}},
\end{aligned}
$$

where $k^{A}, k^{B}$, and $k^{S}$ are the third-order rate coefficients for three possible reaction pathways: $A, B$, and $S .{ }^{1}$ According to the convention introduced earlier, $A$ and $B$ are the two distinct formation pathways of an asymmetric isotopologue of ozone

$$
{ }^{16} \mathrm{O}+{ }^{16} \mathrm{O}^{18} \mathrm{O} \stackrel{A}{\longrightarrow}{ }^{16} \mathrm{O}^{16} \mathrm{O}^{18} \mathrm{O} \stackrel{B}{\longleftarrow}{ }^{16} \mathrm{O}^{16} \mathrm{O}+{ }^{18} \mathrm{O},
$$

whereas the $S$ corresponds to the formation of the symmetric isotopologue of ozone

$$
{ }^{16} \mathrm{O}+{ }^{18} \mathrm{O}^{16} \mathrm{O} \stackrel{S}{\longrightarrow}{ }^{16} \mathrm{O}^{18} \mathrm{O}^{16} \mathrm{O} \stackrel{S}{\longleftarrow}{ }^{16} \mathrm{O}^{18} \mathrm{O}+{ }^{16} \mathrm{O} .
$$

\footnotetext{
a)Present address: Theoretical Division (T-1, MS B221), Los Alamos National Laboratory, Los Alamos, New Mexico 87545, USA.

b) Author to whom correspondence should be addressed: dmitri.babikov@ mu.edu
}

$K_{\mathrm{ex}} \approx 1 / 2$ in Eqs. (1) and (2) is an equilibrium constant for fast exchange of isotopes between the two channels: ${ }^{16} \mathrm{O}$ $+{ }^{16} \mathrm{O}^{18} \mathrm{O} \leftrightarrow{ }^{16} \mathrm{O}^{16} \mathrm{O}+{ }^{18} \mathrm{O}$. Experimental data ${ }^{2}$ indicate that the formation of asymmetric ozone proceeds faster through the deeper channel of pathway $A$ compared to the shallower channel of pathway $B$, by $55 \%$, and that, on average, these two pathways are faster than the reaction $S$, by $16 \%$. Thus, for singly substituted ozone in the experiment, $\zeta_{\exp }=1.55$ and $\eta_{\exp }=1.16$. Similarly, for the formation of the doubly substituted isotopomer of ozone, the reaction pathways are

$$
\begin{aligned}
& { }^{18} \mathrm{O}+{ }^{18} \mathrm{O}{ }^{16} \mathrm{O} \stackrel{A}{\longrightarrow}{ }^{18} \mathrm{O}^{18} \mathrm{O}^{16} \mathrm{O} \stackrel{B}{\longleftarrow}{ }^{18} \mathrm{O}^{18} \mathrm{O}+{ }^{16} \mathrm{O}, \\
& { }^{18} \mathrm{O}+{ }^{16} \mathrm{O}^{18} \mathrm{O} \stackrel{S}{\longrightarrow}{ }^{18} \mathrm{O}^{16} \mathrm{O}^{18} \mathrm{O} \stackrel{S}{\longleftarrow}{ }^{18} \mathrm{O}^{16} \mathrm{O}+{ }^{18} \mathrm{O},
\end{aligned}
$$

where the reaction channel of the pathway $B$ is deeper, the corresponding rate coefficient $k^{B}$ is larger, and $K_{\mathrm{ex}} \approx 2$ for the isotope exchange ${ }^{16} \mathrm{O}+{ }^{18} \mathrm{O}^{18} \mathrm{O} \leftrightarrow{ }^{16} \mathrm{O}^{18} \mathrm{O}+{ }^{18} \mathrm{O}$. For convenience, the definition of the $\zeta$-effect is reversed in this case $\zeta=k^{B} /\left(K_{\mathrm{ex}} k^{A}\right)$. An experimental value of the $\zeta$-effect for the doubly substituted ozone is even larger, $\zeta_{\exp }=1.63$. But, interestingly, the value of the $\eta$-effect is the same as in the singly substituted ozone, within experimental accuracy $\eta_{\exp }=1.16$.

These effects have never been convincingly reproduced by first-principles calculations, although many hypotheses have been proposed by different research groups, and several approximate models have been developed and have already 
shed some light on the possible origin of these effects. ${ }^{3}$ It was shown by several authors that purely classical trajectories do not explain the anomalous large isotope effect in ozone, ${ }^{4-8}$ so one must refer to the quantum mechanical treatment of the process. In particular, the difference of the vibrational zero-point energies $^{2,9-12}$ and the properties of the scattering resonances in the presence of a zero-point energy change ${ }^{13-16}$ seem to be the key to this mystery. Stabilization of the metastable $\mathrm{O}_{3}{ }^{*}$ by bath gas holds some potential for isotopic effects too, but the calculations of energy-transfer carried out so far are inconclusive. ${ }^{17,18}$ The role of electronically non-adiabatic processes, such as spin-orbit coupling, was suggested ${ }^{9}$ but later was ruled out. ${ }^{19}$

Unfortunately, a rigorous quantum mechanical treatment of the entire process of ozone formation is computationally unaffordable, even if we focus on the energy-transfer (Lindemann-Hinshelwood) mechanism of ozone formation: $\mathrm{O}$ $+\mathrm{O}_{2} \Leftrightarrow \mathrm{O}_{3}^{*} \stackrel{+\mathrm{M}}{\longrightarrow} \mathrm{O}_{3}$, neglecting other mechanisms, such as the chaperon mechanism important at lower temperature ${ }^{20}$ or the direct three-body process important at higher pressures. ${ }^{21}$ The "dream" calculation would require an accurate variational description of all vibrational states of ozone, both the bound $\mathrm{O}_{3}$ states below dissociation threshold and the scattering resonances $\mathrm{O}_{3}{ }^{*}$ above it, in a broad range of rotational excitations that correspond to room temperature and taking into account all terms of the Hamiltonian operator, including the Coriolis couplings. For scattering resonances $\mathrm{O}_{3}{ }^{*}$, one needs an accurate quantum description of their formation from and spontaneous decay to $\mathrm{O}+\mathrm{O}_{2}$, but also the inelastic scattering calculations of their quenching onto the stable $\mathrm{O}_{3}$ states and, desirably, their collision-induced dissociation by third-body collisions. All relevant isotopic formation pathways of several isotopologues of ozone should be treated on equal footing, to avoid any bias. The potential energy surface of the adiabatic ground electronic state is sufficient for this problem, but a switch of the electronic state character from being symmetric $\mathrm{X}^{1} \mathrm{~A}_{1}$ in the covalent well region, where ozone is formed, to being anti-symmetric in the $\mathrm{O}_{2}\left({ }^{3} \Sigma_{g}^{-}\right)+\mathrm{O}\left({ }^{3} \mathrm{P}\right)$ asymptote (the geometric phase effect) should be incorporated into the treatment of vibrational states, for example, using the non-Born-Oppenheimer gauge theory. ${ }^{22,23}$

In practice, it is impossible to carry out all these calculations since the PES of ozone is deep and the oxygen nuclei are all heavy, which results in more than three hundred vibrational ${ }^{24-26}$ and many rotational states in the ozone molecule itself, but also requires an unaffordable number of partial waves for the quantum description of scattering of a heavy quencher $\mathrm{M}$, such as $\mathrm{Ar}$ or $\mathrm{N}_{2}$. For this reason, many authors employed approximations, by simplifying either the treatment of the ozone molecule itself (e.g., by dimensional reductions or neglecting its rotation), ${ }^{27,28}$ or the description of the $\mathrm{O}_{3}{ }^{*}+\mathrm{M}$ collision process (e.g., by sudden approximation or strong collision assumption), ${ }^{29,30}$ or by mixing the classical and quantum mechanics. ${ }^{31,32}$

In this work, we still use a basic model for the stabilization step, but to our best knowledge, we offer the most advanced treatment of scattering resonances in ozone. We compute energies, widths (decay/formation rates), and wave functions of resonances using an accurate variational approach and employing the hyper-spherical coordinates that enable rigorous incorporation of symmetry and treat all pathways of ozone formation $(A, B$, and $S)$ on equal footing. Dimensional reduction is avoided, and all degrees of freedom and all vibration modes are taken into account. The full range of hyper-angles is used, which permits simultaneous calculations over three ozone wells, namely, for symmetric ${ }^{16} \mathrm{O}^{18} \mathrm{O}^{16} \mathrm{O}$ and asymmetric ${ }^{16} \mathrm{O}^{16} \mathrm{O}^{18} \mathrm{O}$ in singly substituted ozone, and for symmetric ${ }^{18} \mathrm{O}^{16} \mathrm{O}^{18} \mathrm{O}$ and asymmetric ${ }^{18} \mathrm{O}^{18} \mathrm{O}^{16} \mathrm{O}$ in doubly substituted ozone. A broad range of rotational excitations is covered, up to $J=56$. Direct comparison with experimental data is carried out using the framework developed in Ref. 49. Besides the Coriolis coupling effect ${ }^{33}$ (that is numerically affordable to include for the lightest molecules only) and the geometric phase effect ${ }^{34,35}$ (that is typically small at room temperature), our treatment of scattering resonances is expected to be close to a hypothetic exact calculation.

In our previous paper, ${ }^{49}$ we outlined a theoretical framework for calculating the three rate coefficients in Eqs. (1) and (2), based on the quantum mechanical calculations of scattering resonances, namely, ${ }^{1}$

$$
\begin{aligned}
k^{A}= & \frac{k_{\mathrm{stab}}}{Q^{A}} \sum_{J \Lambda p}(2 J+1) \sum_{i}^{2 N} e^{-E_{i} / k T} \frac{\Gamma_{i}^{A}}{\Gamma_{i}+\left(k_{\mathrm{stab}}+k_{\mathrm{diss}}\right)[\mathrm{M}]}, \\
k^{B}= & \frac{k_{\mathrm{stab}}}{e^{-\Delta \mathrm{ZPE} / k T} Q^{B}} \sum_{J \Lambda p}(2 J+1) \\
& \times \sum_{i}^{2 N} e^{-E_{i} / k T} \frac{\Gamma_{i}^{B}}{\Gamma_{i}+\left(k_{\mathrm{stab}}+k_{\mathrm{diss}}\right)[\mathrm{M}]}, \\
k^{S}= & \frac{k_{\mathrm{stab}}}{Q^{A}} \sum_{J \Lambda p}(2 J+1) \sum_{i}^{N} e^{-E_{i} / k T} \frac{\Gamma_{i}}{\Gamma_{i}+\left(k_{\mathrm{stab}}+k_{\mathrm{diss}}\right)[\mathrm{M}]} .
\end{aligned}
$$

Input data for these expressions are energies $E_{i}$ and widths $\Gamma_{i}$ (spontaneous decay rate coefficients) of individual resonances labeled by index $i$, computed for the range of rotational excitations described by quantum numbers $J$ and $\Lambda$ (thermal distribution), and parity $p$. Differences between the two pathways, $A$ and $B$, are accounted in these equations by the ro-vibrational zero-point energy change $\triangle \mathrm{ZPE}$, two partition functions $Q^{A}$ and $Q^{B}$ of $\mathrm{O}+\mathrm{O}_{2}$ reagents, and the channelspecific decay rate coefficients $\Gamma_{i}^{A}$ and $\Gamma_{i}^{B}$. Stabilization and dissociation rate coefficients $k_{\text {stab }}$ and $k_{\text {diss }}$ are taken the same for all resonances, for transparency.

Several moieties in Eqs. (3)-(5) may, potentially, lead to the isotope effects in Eqs. (1) and (2). The role of molecular symmetry and the mass-effect can be determined using what we call the zeroth and first levels of theory, respectively. ${ }^{49}$ Predictions of such approximate theoretical treatments will be reported elsewhere. They indicate that no anomalous isotope effects should come from symmetry considerations, if all factors are taken into account properly, which is not trivial, since large symmetry-related factors, such as $1 / 2$ and 2 , appear in seven (!) different places in the formalism. It can also be shown that masses of isotopic substitutions create appreciable differences in vibrational and rotational energies, but, interestingly, these play against each other and largely cancel, creating only a small isotope effect, insufficient to explain experimental observations. These relevant findings 
TABLE I. Two isotope effects in singly and doubly substituted ozone molecules, computed at different levels of theory and measured in the experiment.

\begin{tabular}{|c|c|c|c|c|c|}
\hline \multicolumn{2}{|r|}{ Theory } & \multicolumn{2}{|c|}{$\zeta$-Effect } & \multicolumn{2}{|c|}{$\eta$-Effect } \\
\hline Level & Model/meaning & Single & Double & Single & Double \\
\hline Oth & Symmetry effect only & 1.00 & 1.00 & 1.00 & 1.00 \\
\hline $1 \mathrm{st}$ & Mass-effect (aka statistical) & 1.13 & 1.13 & 0.95 & 1.04 \\
\hline 2nd & Shape resonances only & 0.87 & 1.39 & 0.73 & 0.73 \\
\hline $3 r d$ & Feshbach resonances enabled & 1.49 & 1.38 & 1.03 & 1.12 \\
\hline & Experiment $^{2}$ & 1.55 & 1.63 & 1.16 & 1.16 \\
\hline
\end{tabular}

are listed in the first two rows of Table I. Both zeroth and first levels of theory are rather approximate, and it can be demonstrated that the first level is equivalent to the statistical treatment.

In this paper, we go further and take into account the properties of quantized resonances, by computing their energies and lifetimes. In general, we distinguish two types of scattering resonances, called "shape" resonances and Feshbach resonances. ${ }^{36,37}$ Shape resonances are trapped behind the centrifugal barrier and are populated by tunneling. Since tunneling is a purely quantum phenomenon, not present in the classical trajectory simulations, one may wonder whether quantum tunneling, through shape resonances, can be responsible for the anomalously large isotope effects? This question has never been addressed in the literature and is addressed here. The Feshbach resonances are populated by coupling between the vibrational channels in the system, which can also be viewed as a vibrationally non-adiabatic quantum phenomenon. ${ }^{38}$

Thus, in this paper, we add two more levels of theory to Table I. At the second theory level, we disregard couplings between the diabatic vibrational channels in the problem, in order to disable the formation of Feshbach resonances and study the shape resonances alone. This level of theory is also approximate, devised solely to determine the role of quantum tunneling in the ozone formation process and in the isotope effects. At the third level of theory, we include the couplings between the channels and determine the role of Feshbach resonances. This is the most accurate theory level of this work, which also incorporates interactions between the reaction pathways $A$ and $B$ (in the region of the deep covalent well on the PES) and between the pathways $A$ and $S$ (in the entrance/exit channels on the global PES of ozone). The goal of developing this hierarchy of theory levels is to identify the physical origin of the unusual isotope effects seen in the experiment, rather than just to reproduce experiment by an all-inclusive calculation.

\section{THEORY}

\section{A. Sequential diagonalization truncation}

All details of our method can be found in the recently published detailed papers. ${ }^{24,39}$ Here we recap, briefly, only those elements of the theory that are needed to explain the essence of new calculations.
Vibrational motion of oxygen atoms in the ozone molecule is described using hyper-spherical coordinates $\rho, \theta$, and $\varphi$. The hyperradius $\rho$ plays the role of the reaction coordinate, while the hyper-angles $\theta$ and $\varphi$ describe the internal motion. The Hamiltonian operator separates accordingly, ${ }^{24}$ $\hat{H}=\hat{T}_{\rho}+\hat{T}_{\theta \varphi}+V(\rho, \theta, \varphi)$, so the calculation of vibrational states can be done sequentially. First, a two-dimensional bound-state problem is solved for the motion along $\theta$ and $\varphi$ to determine wave functions $\psi_{k}(\theta, \varphi)$ and energies $\varepsilon_{k}$ for slices of the PES, at different values of $\rho$. For the slice number $n$, the two-dimensional Schrodinger equation is $^{24}$

$$
\hat{h}^{n} \psi_{k}^{n}(\theta, \varphi)=\varepsilon_{k}^{n} \psi_{k}^{n}(\theta, \varphi),
$$

where $\hat{h}^{n}=\hat{T}_{\theta \varphi}+V^{n}(\theta, \varphi)$ is the Hamiltonian operator for hyper-angles, with $V^{n}(\theta, \varphi)$ representing a two-dimensional slice through $\theta$ and $\varphi$ of the potential energy surface $V(\rho, \theta, \varphi)$ at a fixed value of $\rho=\rho^{n}$. Couplings between the different values $\rho^{n}$ of the grid are contained in the overlap matrix $^{24}$

$$
O_{k l}^{n m}=\left\langle\psi_{l}^{m} \mid \psi_{k}^{n}\right\rangle
$$

These couplings are taken into account at the next step of calculations, when the 2D-wave functions $\psi_{k}^{n}(\theta, \varphi)$ at all slices $\rho^{n}$ are collected and used to form an efficient (locally optimal) basis for the representation of the overall 3D Hamiltonian matrix $^{40,41}$

$$
H_{k l}^{n m}=O_{k l}^{n m} \times T^{n m}+\delta_{k l} \delta_{n m} \varepsilon_{k}^{n} .
$$

Here $T^{n m}$ is a matrix of the kinetic energy operator $\hat{T}_{\rho}$ in the DVR basis (discrete variable representation, which is a grid of $N$ points $\rho^{n}$ ). The block-structure of the Hamiltonian matrix $H_{k l}^{n m}$ was discussed in detail in Ref. 24. It has the same size as $O_{k l}^{n m}$ and is obtained from it by multiplying each of its $\rho$-blocks by the corresponding element of the matrix $T^{n m}$ and then adding to each diagonal element the corresponding value of two-dimensional energy $\varepsilon_{k}^{n}$.

Diagonalization of $H_{k l}^{n m}$ gives energies $E_{i}$, widths $\Gamma_{i}$ (if the complex absorbing potential, CAP, is added to the PES), ${ }^{39}$ and wave functions $\Psi_{i}(\rho, \theta, \varphi)$ of the $3 \mathrm{D}$-states, and typically, all authors proceed straight to that final step. We will, eventually, do that too, but before, we will also formulate and test a useful approximation to the problem, in which some couplings are neglected, based on the physical meaning of the two-dimensional states $\psi_{k}(\theta, \varphi)$. These states carry useful information for the interpretation of the results and for the analysis of the isotope effects.

\section{B. Diabatization of ro-vibrational channels}

It is relatively easy to tell, just by looking at the localization of wave functions $\psi_{k}(\theta, \varphi)$ near the transition state point $\left(\rho \approx \rho^{\ddagger}\right.$ ), what reaction pathway they correspond to $A, B$, or $S$. From there, one can move to the left $\left(\rho<\rho^{\ddagger}\right)$ and right $\left(\rho>\rho^{\ddagger}\right)$ connecting the $\psi_{l}^{m}(\theta, \varphi)$ states in a new slice with those in the previous slice $\psi_{k}^{n}(\theta, \varphi)$ diabatically, based on the magnitudes of their overlaps $O_{k l}^{n m}$. This procedure works reasonably well for several lower diabatic vibrational channels, where the density of states is not high. It effectively splits these states onto three groups, those that 

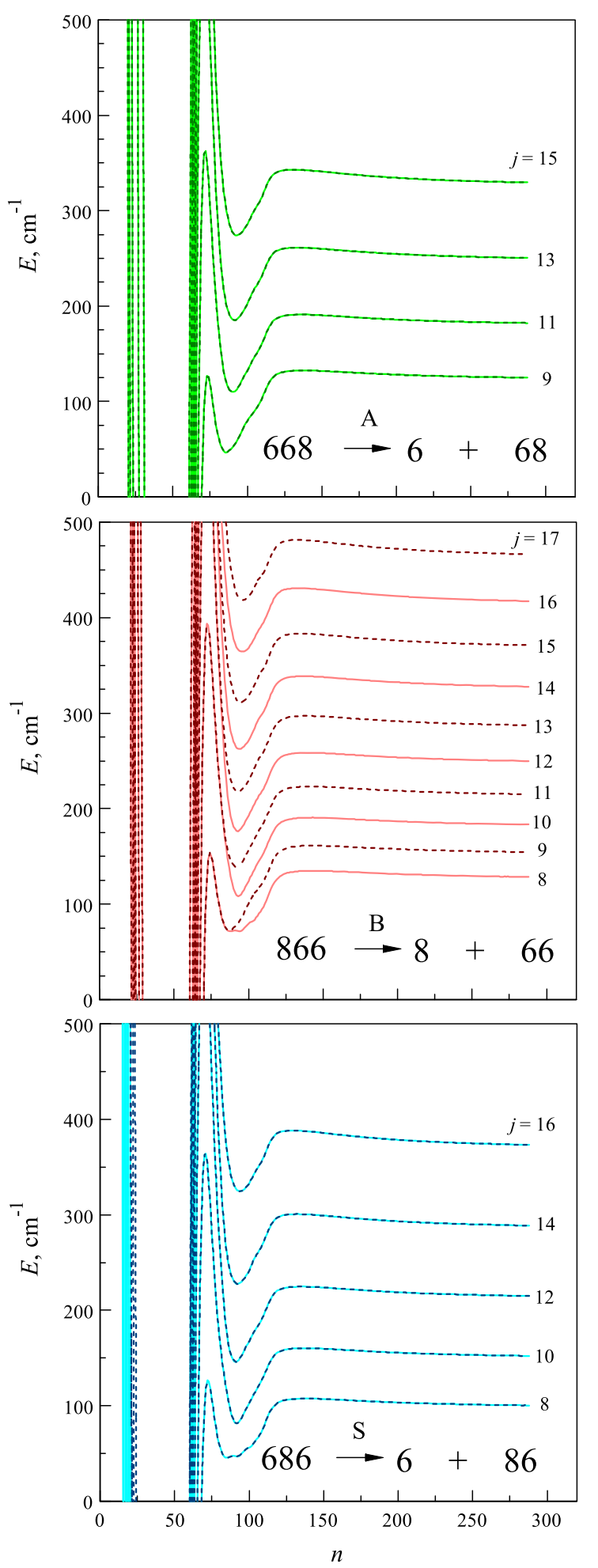

FIG. 1. Energies of diabatic vibrational states computed along the reaction coordinate $\rho$ for the singly substituted isotopomer of ozone with typical rotational excitation $(J=24, \Lambda=8)$. Three frames correspond to three pathways of ozone formation: $A, B$, and $S$. A connection to the asymptotic rotational states of $\mathrm{O}_{2}$ is emphasized. Solid and dashed curves correspond to vibrational state symmetries $A_{1}$ and $B_{1}$, respectively. They are nearly identical in the pathway $A$ and in the asymptotic region of pathway $S$. In the pathway $B$, they go to different asymptotes.

correspond to the reaction pathways $A, B$, and $S$. Figure 1 gives diabatic curves $\varepsilon_{k}(\rho)$ obtained in this way, for the reaction pathways $A, B$, and $S$ of singly substituted ozone, plotted separately in three frames of the figure. From Fig. 1, we can see that in the limit $\rho \rightarrow \infty$, the diabatic curves $\varepsilon_{k}(\rho)$ correlate with rotational states of $\mathrm{O}_{2}$ reagents in the corresponding entrance channels. In the range of small $\rho$, they describe vibrations in the corresponding covalent wells, where $\mathrm{O}_{3}$ is formed.

Based on this analysis, one can propose approximation in which the couplings (overlaps $O_{k l}^{n m}$ ) between different diabatic channels are neglected. This effectively splits the Hamiltonian matrix of Eq. (8) onto many independent small matrixes of the size $N \times N$ (where $N$ is the number of grid points $\rho^{n}$ along the hyperradius; see above). These can be diagonalized separately with small numerical effort. Since each such matrix includes only one diabatic state, indexes $k$ and $l$ used in Eq. (8) are not needed, and the structure of each matrix can be expressed as

$$
H^{n m}=O^{n m} \times T^{n m}+\delta_{n m} \varepsilon^{n} .
$$

This simple formula contains, as usual, the diagonal matrix of the potential energy operator and the full matrix of the kinetic energy operator. Physically, this corresponds to an independent treatment of each potential curve in Fig. 1 or to a large number of quasi-1D problems solved along $\rho$ for independent diabatic vibrational states. This seems to be a significant simplification since many couplings between the vibrational states are neglected. However, one should remember that the largest couplings in the problem are still retained, since the diabatic states are employed (rather than adiabatic), and those are connected by the largest overlaps $O_{k l}^{n m}$, by construct.

We want to emphasize that the goal of this simplifying assumption is not to come out with an approximate theory that may give a reasonably accurate description of ozone (although similar approximations are sometimes employed ${ }^{42}$ ). Instead, this diabatic approximation is used to disable the mechanism for the formation of Feshbach resonances, by neglecting the vibrational couplings and making independent the individual vibrational channels. In this case, the reaction mechanism is artificially restricted to the formation of shape resonances only, populated exclusively by tunneling through the centrifugal barriers of the individual diabatic vibrational curves in Fig. 1. In this way, the role of quantum tunneling in the ozone formation process is elucidated, and its contribution to the isotope effects is determined (at the 2nd level of theory), separately from other competing processes. The role of vibrational channel couplings, or Feshbach resonances, is incorporated later (at the 3rd level of theory).

\section{Connection to the statistical approach}

Equations (3)-(5) of the Introduction can also be simplified, if one follows the approximate treatment of scattering resonances outlined in Sec. II B. Importantly, this simplification permits to define a quantum-dynamical analog of the statistical partition function, which is convenient for the comparison of the results obtained here with the results of a simplified treatment of the process using a statistical approach (1st level of theory). ${ }^{49}$ Namely, one can rewrite Eqs. (3)-(5) in the following 
form:

$$
\begin{aligned}
k^{A} & =\frac{k_{\mathrm{stab}}}{Q_{\mathrm{O}+\mathrm{O}_{2}}^{A}} \sum_{J \Lambda}(2 J+1) e^{-E_{A}^{\ddagger} / k T} \tilde{Q}_{\mathrm{O}_{3}^{*}}^{A}, \\
k^{B} & =\frac{k_{\mathrm{stab}}}{Q_{\mathrm{O}+\mathrm{O}_{2}}^{B}} \sum_{J \Lambda}(2 J+1) e^{-E_{B}^{\ddagger} / k T} \tilde{Q}_{\mathrm{O}_{3}^{*}}^{B}, \\
k^{S} & =\frac{k_{\mathrm{stab}}}{Q_{\mathrm{O}+\mathrm{O}_{2}}^{A}} \sum_{J \Lambda}(2 J+1) e^{-E_{S}^{\ddagger} / k T} \tilde{Q}_{\mathrm{O}_{3}^{*}}^{S},
\end{aligned}
$$

where for the metastable ozone states in each pathway $A, B$, and $S$ (represented by scattering resonances), we introduced the dynamical partition function $\tilde{Q}_{\mathrm{O}_{3}^{*}}$ and the activation energy $E^{\ddagger}$ needed to reach these metastable states, starting at the asymptotic value of energy for each pathway. Namely, for the pathways $A$ and $S$, we set $E^{\ddagger}=\varepsilon_{0}^{\ddagger}$, where $\varepsilon_{0}^{\ddagger}=\varepsilon_{0}\left(\rho^{\ddagger}\right)$ is the energy of the ground diabatic state at the position of centrifugal barrier $\rho^{\ddagger}$. For a shallower channel of the pathway $B$, we set $E^{\ddagger}=\varepsilon_{0}^{\ddagger}-\Delta \mathrm{ZPE}$, so the factor $e^{-\Delta \mathrm{ZPE} / k T}$ in Eq. (4) cancels. Summation over two parities $p$ in Eqs. (3)-(5) is absorbed by the partition function

$$
\tilde{Q}_{\mathrm{O}_{3}^{*}}=\sum_{p} \sum_{k} e^{-\left(\varepsilon_{k}^{\ddagger}-\varepsilon_{0}^{\ddagger}\right) / k T} \sum_{i} e^{-\delta E_{i} / k T} w\left(\Gamma_{i}\right),
$$

where index $k$ labels the diabatic vibrational channels, while index $i$ labels resonances within each channel. Note that energies $E_{i}$ of shape resonances, computed at the 2 nd level of theory, can be measured relative to the barrier top $\varepsilon_{k}^{\ddagger}=\varepsilon_{k}\left(\rho^{\ddagger}\right)$ of each independent diabatic curve. So, for convenience, we introduced $\delta E_{i}=E_{i}-\varepsilon_{k}^{\ddagger}$, to represent the deviation of resonance energy from the top of its barrier, which can be both negative (for under the barrier) and positive (for over the barrier resonances).

The weight $w$ introduced in Eq. (13) describes the importance of given resonance for the recombination process and is determined by its decay rate coefficient $\Gamma_{i}$ (the resonance width),

$$
w\left(\Gamma_{i}\right)=\frac{\Gamma_{i}}{\Gamma_{i}+\left(k_{\mathrm{stab}}+k_{\mathrm{diss}}\right)[\mathrm{M}]} .
$$

In the limit $\Gamma_{i}>>\left(k_{\mathrm{stab}}+k_{\mathrm{diss}}\right)[\mathrm{M}]$, we obtain a constant limiting value $w \rightarrow 1$, which corresponds to broad resonances and/or a low-pressure regime. In the opposite limit (which corresponds to narrow resonances and/or high-pressure regime), we see that $w \rightarrow 0$ as a linear function of $\Gamma_{i}$. Thus, the narrower (longer-lived) resonances contribute less to recombination at a given pressure. If the diabatic approximation of Sec. II B is employed, then resonance widths $\Gamma_{i}$ are computed separately for the pathways $A, B$, and $S$, simply because all vibrational channels are made independent.

It is instructive to show that, under certain conditions, formula (13) for the dynamic partition function $\tilde{Q}_{\mathrm{O}_{3}^{*}}$ transforms into a standard formula for the statistical partition function introduced at the transition state. ${ }^{49}$ Namely, if one assumes that there is only one resonance per diabatic channel (no summation over $i)$, its energy is exactly at the barrier top $\left(\delta E_{i}=0\right)$, and its width is large $(w=1)$, then

$$
Q_{T S}=\sum_{p} \sum_{k} e^{-\left(\varepsilon_{k}^{*}-\varepsilon_{0}^{*}\right) / k T} .
$$

It appears that Eqs. (10)-(12) can also be employed in the most rigorous coupled-channel calculations of Feshbach resonances, with all couplings included, all vibrational channels coupled, and all the reaction pathways interacting (3rd level of theory in the hierarchy). For this, each resonance of the asymmetric ozone has to be included into both $\tilde{Q}_{\mathrm{O}_{3}^{*}}^{A}$ and $\tilde{Q}_{\mathrm{O}_{3}^{*}}^{B}$ but with different values of $\delta E_{i}=E_{i}-\varepsilon_{0}^{\ddagger}$ (relative to the transition state of each pathway) and with specific decay rate coefficients $\Gamma_{i}^{A}$ and $\Gamma_{i}^{B}$ in the numerator of Eq. (14), determined by splitting the total decay rate coefficient $\Gamma_{i}$ as it was explained elsewhere. ${ }^{1}$ In this case, the resonance energies $E_{i}$ can only be measured relative to the barrier top of the ground diabatic state $\varepsilon_{0}^{\ddagger}$ in each pathway. Then, the analog of Eq. (13) is

$$
\begin{aligned}
\tilde{Q}_{\mathrm{O}_{3}^{*}} & =\sum_{p} \sum_{i} e^{-\left(E_{i}-\varepsilon_{0}^{*}\right) / k T} w\left(\Gamma_{i}\right) \\
& =\sum_{p} \sum_{i} e^{-\delta E_{i} / k T} w\left(\Gamma_{i}\right) .
\end{aligned}
$$

As explained above, the definitions and the meanings of $\delta E_{i}$ in Eqs. (13) and (15) are slightly different.

It should also be recognized that different resonances may exhibit drastically different properties. In particular, very broad resonances (scattering states) exhibit significant delocalization of the wave function over the PES. For such states, only a small part of the wave function is located within the covalent well. It was shown in our earlier work ${ }^{43}$ that only the states localized over the well can be efficiently stabilized. Cross sections for the stabilization of states located outside of the covalent well (such as van-der-Waals states) are negligibly small. Consequently, for delocalized resonances, the rate coefficient for stabilization $k_{\text {stab }}$ should be scaled down, taking into account the probability of finding the system inside of the covalent well region. To implement this feature, we compute this probability, $P^{\text {well }}$, using the wave function of each resonance and set the stabilization rate coefficient as a product, $k_{\mathrm{stab}} P^{\text {well }}$. The value of $k_{\text {stab }}$ is the same for all resonances, based on $\sigma_{\text {stab }}=154 a_{0}^{2}$ adopted from Ref. 18. The value of $P_{i}^{\text {well }}$ is different for different resonances, so it is more convenient to absorb it by the partition function. For example, instead of Eq. (15), we can write

$$
\tilde{Q}_{\mathrm{O}_{3}^{*}}=\sum_{p} \sum_{i} e^{-\delta E_{i} / k T} w\left(\Gamma_{i}\right) P_{i}^{\mathrm{well}} .
$$

And the same for Eq. (13). We want to emphasize one more time that this moiety is not a statistical partition function, but rather is its dynamical analog. Besides the standard statistical Boltzmann factor that depends on resonance energies $E_{i}$, this expression also includes information about the formation/decay rates of resonances (through $\Gamma_{i}$ ) and their stabilization probabilities (through $\left.P_{i}^{\text {well }}\right)$.

Careful convergence studies with respect to the extent of the grid in $\rho$, the density of grid points, and the parameters of the complex absorbing potential have been conducted and reported in our earlier detailed work. ${ }^{24,39}$ 


\section{RESULTS AND DISCUSSION}

\section{A. Shape resonances}

For the lower theory level of this paper (2nd, with diabatic vibrational channels treated independently), we carried out calculations of resonances on a dense grid of rotational excitation quantum numbers $J$ and $\Lambda$, namely, $4 \leq J \leq 56$ with a step of four and $0 \leq \Lambda \leq J$ with a step of two. The dependence of the dynamical partition function $\tilde{Q}$ on the rotational excitations is presented in Fig. 2 for the pathway $B$ of singly substituted ozone (and in the supplementary material for all other cases, see Figs. S1 and S2). It should be mentioned that a simplified theoretical treatment of this process, equivalent to the statistical description (to be reported elsewhere ${ }^{49}$ ), predicts the same value of the partition function at the transition state of both singly substituted and doubly substituted ozone, $Q \sim 2.8$ at room temperature, which also is insensitive to the rotational excitations $J$ and $\Lambda$. In contrast to that statistical behavior, we see from Fig. 2 and Figs. S1 and S2 that the dynamical partition function $\tilde{Q}$ varies significantly as a function of $J$ and $\Lambda$. For example, several values of $\tilde{Q}$ close to 4 can be found in Fig. 2 and Figs. S1 and S2, for extreme values of rotational excitations $J$ and $\Lambda$. The values of $\tilde{Q}$ in the range 2-to-3 are more typical and can be found along the "ridges" that dominate the landscape of Fig. 2 and Figs. S1 and S2. However, for the majority of rotational excitations, the value of $\tilde{Q}$ is less than 1 . Consequently, this level of theory leads to lower values of rate coefficients, relative to the statistical description with the partition function computed at the transition state. ${ }^{49}$ Recombination rate coefficients for different reaction pathways, for singly and doubly substituted ozone molecules, are collected in Table II.

The origin of pronounced "ridges" in Fig. 2 and Figs. $\mathrm{S} 1$ and $\mathrm{S} 2$ is worth exploring. We found that each such ridge corresponds to appearance, at energy near the top of the centrifugal barrier, of a particular vibrational state lifted by the rotational excitation. Indeed, shape resonances can be thought of as bound vibrational states lifted by the centrifugal potential up, to energies above the dissociation threshold, where they can be populated by tunneling through the centrifugal

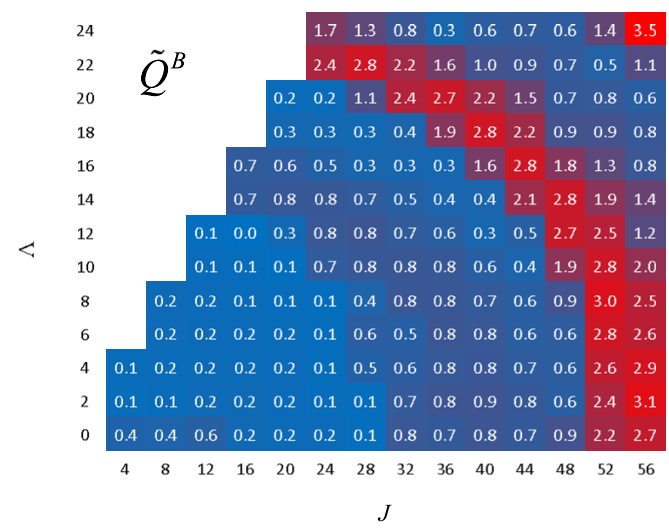

FIG. 2. Dynamical partition function, as defined by Eq. (13), for the pathway $B$ of singly substituted ozone. The dependence on two quantum numbers of rotational excitation $J$ and $\Lambda$ (plotted along horizontal and vertical axes, respectively) is computed using the 2 nd level of theory to determine the contribution of shape resonances.
TABLE II. Recombination rate coefficients (third order, $10^{-35} \mathrm{~cm}^{6} / \mathrm{s}$ ) for three different reaction pathways of two isotopomers of ozone computed at different levels of theory.

\begin{tabular}{lccccccc}
\hline \hline & \multicolumn{3}{c}{ Singly substituted $\mathrm{O}_{3}$} & & \multicolumn{3}{c}{ Doubly substituted $\mathrm{O}_{3}$} \\
\cline { 2 - 3 } Theory level & $k^{A}$ & $k^{B}$ & $k^{S}$ & & $k^{A}$ & $k^{B}$ & $k^{S}$ \\
\hline 1st & 6.5 & 12 & 6.5 & & 6.0 & 13 & 6.1 \\
2nd & 1.1 & 2.5 & 1.6 & & 0.9 & 2.6 & 1.6 \\
3rd & 38 & 51 & 31 & & 29 & 78 & 31 \\
Experiment $^{2}$ & 43 & 55 & 30 & & 28 & 90 & 31 \\
\hline \hline
\end{tabular}

barrier. Since oxygen atoms are heavy, the tunneling is relatively weak, and the process of population/decay becomes efficient only when the resonance approaches the barrier top. The necessary lift can be achieved by various combinations of $J$ and $\Lambda$ values. For example, we took a close look at six different $(J, \Lambda)$-pairs along the major ridge in Fig. 2: $(J, \Lambda)$ $=(28,22),(32,20),(36,18),(40,16),(44,14)$, and $(48,12)$. For each case, we identified a resonance that makes dominant contribution (typically, there is only one such resonance) and plotted its wave function along the hyper-radial coordinate $\rho$, in Fig. 3. From this figure, we see that their vibrational wave functions are almost identical, despite the fact that their rotational excitations are very different. They all correspond to 10 quanta of vibrational excitation along the hyperradius $\rho$, within the region of the covalent well on the PES of ozone $\left(\rho<5 a_{0}\right)$.

Further analysis showed that the other ridge, in the upper right corner of Fig. 2, corresponds to the vibrational state with 9 quanta of excitation along $\rho$. These two pronounced ridges, and the corresponding vibrational states with 9 and 10 quanta of excitation, were determined to be associated with the ground diabatic channel of the system (labeled above by $\varepsilon_{0}$ ). The third less visible ridge in Fig. 2 was found to be associated with the first excited diabatic channel $\left(\varepsilon_{1}\right)$ and be described by the vibrational state with 9 excitation quanta. Upper diabatic channels ( $\varepsilon_{2}$ and higher) were found to contribute much less to the magnitude of $\tilde{Q}$, at this level of theory.

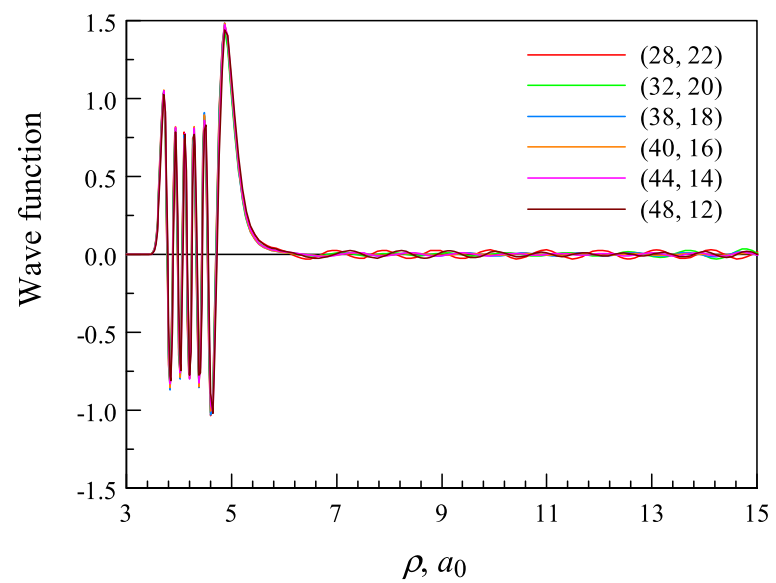

FIG. 3. Wave functions of dominant shape resonances for six different values of rotational excitation chosen along the "ridge" in Fig. 2 (see text). All wave functions indicate ten quanta of vibrational excitation along $\rho$ and overlap nearly perfectly in the region over the covalent well, $\rho<5 a_{0}$. 
This behavior appears to be typical for all pathways $(A, B$, and $S$ ) of both isotopomers (singly and doubly substituted) as illustrated by six frames of Figs. S1 and S2. In all cases, we found that the right-most ridge is due to the vibrational state with 8 or 9 excitation quanta in the ground diabatic channel. The central ridge is due to 9 to 10 excitation quanta in the ground diabatic channel. The left-most ridge is due to 7 to 9 excitation quanta in the first excited diabatic channel. Our conclusion is that only a small number of vibrational states (three to four per reaction pathway) dominate the formation of shape resonances in ozone. Other vibrational states are also present and are included in our calculations, but their contributions are much smaller due to either deep submersion under the centrifugal barrier (that results in small resonance width $\Gamma_{i}$ ) or due to the delocalization of the wave function at energies well above the barrier (that results in small probability in the covalent well $\left.P_{i}^{\text {well }}\right)$. Both cases lead to negligible contribution to the recombination process. We found that it is sufficient to consider only shape resonance within the range $-200<\delta E<+300 \mathrm{~cm}^{-1}$ from each centrifugal barrier. Properties of these resonances, such as the distribution of their lifetimes, were reported elsewhere. ${ }^{1}$

As for isotopic dependencies, we found that this level of theory (2nd level, shape resonances) does not produce any isotope effect that would look like experiment. In particular, the values of $\zeta$ for singly and doubly substituted isotopomers are 0.87 and 1.39 , which means that they deviate from 1.0 in opposite directions, in contrast to experimental data (see Table I). Moreover, the value of $\eta$ is 0.73 for both singly and doubly substituted isotopomers, which is less than 1.0 and, again, is opposite to the experiment (see Table I). Based on these data, we conclude that shape resonances are not responsible for the isotope effects seen in the experiment.

Just to note that these values of $\zeta$ and $\eta$, and all other data reported in Tables I-III, were obtained by excluding from analysis the states with unphysically large values of $\Gamma_{i}$ (exceeding the vibration frequency along $\rho$ at a given energy). We found that wave functions of such states are located outside of the covalent well, between the centrifugal barrier and the CAP. They are artifacts of the method, occurring due to the employment of a finite grid and irrelevant to the recombination process. We also excluded the states with negligible probability in the covalent well $\left(P_{i}^{\text {well }}<0.01\right)$ to avoid delocalized states at high energies that may not be converged. Their influence on isotope effects was checked and found to be insignificant.

\section{B. Feshbach resonances}

At the highest theory level (3rd, with vibrational couplings included), we carried out the calculations of Feshbach resonances in the same range of quantum numbers $J$ and $\Lambda$ and with same increments as in Sec. III A. However, in order to ease calculations, we limited the window of resonance energies by $800 \mathrm{~cm}^{-1}$ above dissociation threshold. This value, equal to $4 k T$ at room temperature, is well sufficient for the calculation of thermal recombination rate coefficients. The dependence of the dynamic partition function $\tilde{Q}$ on rotational excitations $J$ and $\Lambda$ is presented in Fig. 4 for the pathway $B$

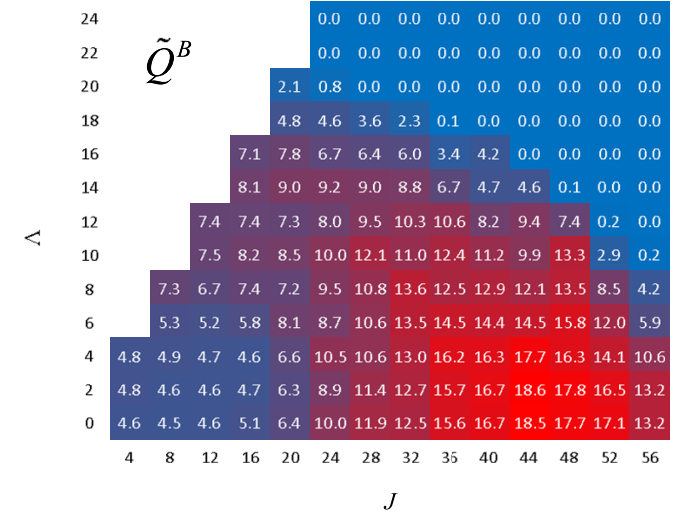

FIG. 4. Dynamical partition function, as defined by Eq. (15), for the pathway $B$ of singly substituted ozone computed using the 3rd level of theory to determine the effect of Feshbach resonances. This dependence can be compared with the contribution of shape-resonances presented in Fig. 2.

of singly substituted ozone (and in the supplementary material for all other cases; see Figs. S5 and S6). In these pictures, all dependencies are smooth, with no sharp maxima or any pronounced ridges. For low rotational excitations, the values of $\tilde{Q}$ are somewhat smaller (see Fig. 4). We found that in those cases, the top of the centrifugal barrier of the ground channel $\varepsilon_{0}^{\ddagger}$ is slightly submerged under the asymptote, which leads to the mixing of vibrational states of the main (covalent) well of ozone with weakly bound states of the van der Waals complex $\mathrm{O} \cdots \mathrm{O}_{2} \cdot{ }^{11}$ The stabilization probability of such highly delocalized states is assumed to be smaller (as determined here by $P_{i}^{\text {well }}$ ) which results in smaller values of $\tilde{Q}$. For extreme rotational excitations, in the upper right corner of Fig. 4, the decrease of $\tilde{Q}$ to basically zero is due to the window of $800 \mathrm{~cm}^{-1}$ enforced in these calculations (in order to make calculations manageable). At these large values of $J$ and $\Lambda$, the height of the centrifugal barrier is above $800 \mathrm{~cm}^{-1}$, which anyway shuts off the recombination reaction due to the Boltzmann factor in Eqs. (9)-(11). Thus, no calculations were done there.

The smooth behavior of $\tilde{Q}(J, \Lambda)$ is quite different from non-regular behavior dominated by "ridges" reported in Fig. 2 (due to the shape-resonances). The reason for this difference is that Feshbach resonances occur more frequently in the spectrum and not just in the proximity of the centrifugal barrier top but also through a wider range of energies above the barrier. It appears that for every rotational state $(J, \Lambda)$, there are many resonances contributing to the recombination process. Typical values of $\tilde{Q}$, summed over two parities, are on the order of 10-to-15 and approach 20 for some rotational states. This is higher than the contribution of shape resonances and is even higher than predicted by statistical description at the 1 st level of theory, where a constant value $Q \sim 2.8$ was obtained. ${ }^{49}$ These data demonstrate the dominant role of Feshbach-type resonances (over shape-type resonances and over statistical description) in the process of ozone formation. In particular, the contribution of Feshbach resonances exceeds that of "shape" resonances by at least an order of magnitude, which makes sense for ozone, where all atoms are heavy and the tunneling is weak. On a qualitative level, this conclusion is very likely to hold in any coordinate system chosen to represent the 
vibrational channels and describe quantum tunneling through the centrifugal barrier.

The values of recombination rate coefficients at this level of theory (for different reaction pathways $k^{A}, k^{B}$, and $k^{S}$ of singly and doubly substituted ozone molecules) are all included in Table II. We see that their absolute values overshoot predictions of theory levels 1st and 2nd, roughly, by factors of $\times 6$ and $\times 20$, respectively, which follows the trend of partition function values for three theory levels, as discussed above. Predictions of the 3 rd (our best) theory level come rather close to the results of experiments.

Just to note that, computing the absolute values of thermal rate coefficients, we excluded from consideration all antisymmetric vibrational states (symmetry $\mathrm{B}_{1}$ ) for rotational excitations with $\Lambda=0$ (for all $J$ values) since in those cases only rotational states of even parity are possible and they would combine with symmetric vibrational states only (symmetry $\left.\mathrm{A}_{1}\right) .{ }^{49}$ This modification had some effect on the absolute values of rate coefficients, decreasing them by $\sim 6 \%$, but had no any appreciable influence on the isotopic effects (see below).

For completeness, we also computed dependencies of rate coefficients on rotational excitation: $k^{A}(J, \Lambda), k^{B}(J, \Lambda)$, and $k^{S}(J, \Lambda)$, which correspond to different terms in the summations of Eqs. (9)-(11), respectively. These extensive data computed for singly and doubly substituted ozone molecules at 2nd and 3rd levels of theory are included in Figs. S3 and S4 and Figs. S7 and S8 of the supplementary material, respectively. One can see that the largest contributions of Feshbach resonances into the recombination process occur near $12 \leq J \leq 40$ and $0 \leq \Lambda \leq 12$, consistent with our earlier results ${ }^{16}$ for unsubstituted ${ }^{16} \mathrm{O}^{16} \mathrm{O}^{16} \mathrm{O}$. Our present calculations cover this range of rotational excitations even better.

In Fig. 5, we go even further into detail and plot the contribution of each individual resonance into the recombination

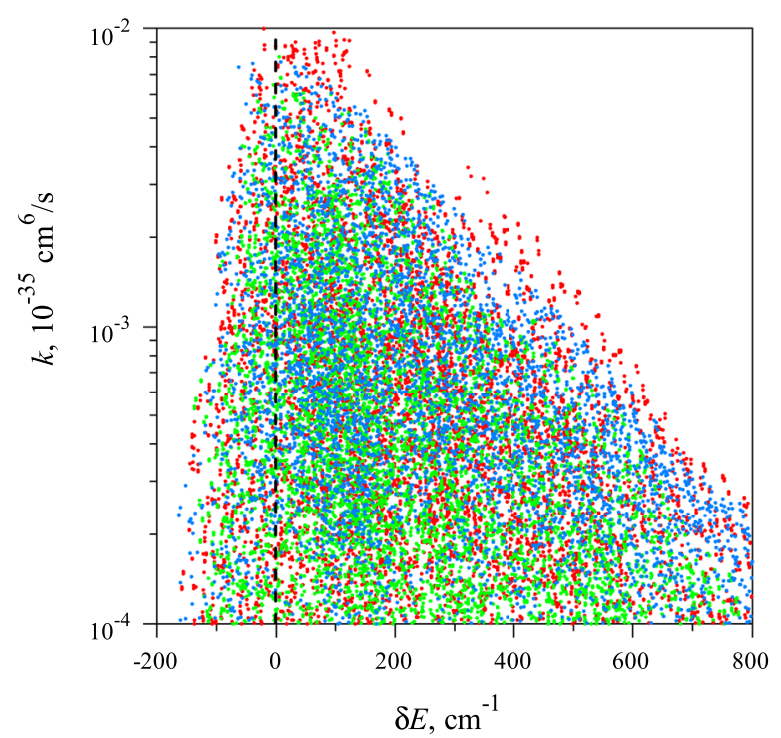

FIG. 5. Contributions of individual resonances into recombination rate coefficients for singly substituted ozone. Green, red, and blue colors correspond to $k^{A}, k^{B}$, and $k^{S}$, respectively (three recombination pathways). These data were computed at the 3rd level of theory, which enables Feshbach resonances. For clarity, only important states are included, with $P^{\text {well }}>0.05$. process, for the case of singly substituted ozone (the case of doubly substituted ozone looks similar). Pathways $A, B$, and $S$ are color-coded in Fig. 5, as before. We see that Feshbach resonances at energies $-150<\delta E<+800 \mathrm{~cm}^{-1}$ make substantial contributions to the recombination process. This range of resonance energies is broader than the range of shape resonances (see above) because Feshbach resonances are populated by couplings between vibrational channels, rather than by tunneling through the barrier. Note, however, that in this figure, energy is reported relative to the barrier top of the lowest vibrational channel, $\varepsilon_{0}^{\ddagger}$ (in each pathway), that can even be slightly submerged at lower rotational excitations. As for the widths of Feshbach resonances, we found that widths in the range $5 \times 10^{-4}<\Gamma<30 \mathrm{~cm}^{-1}$ appear to be most important for the recombination process, very similar to shape-resonances. Majority of these resonances are rather narrow and well separated (non-overlapping), so the assumption of independent resonance states is justified for the kinetics of ozone formation. Additional discussion of resonance properties, such as the dependence of their lifetimes on other physical variables in the problem, was reported elsewhere. ${ }^{1}$

\section{Isotope effects}

For the description of the isotopic $\zeta$-effect in asymmetric ozone molecules, the difference of resonance decay rates into pathways $A$ and $B$ is fundamentally important. The values of $\Gamma_{i}^{A}$ and $\Gamma_{i}^{B}$ are expected to be sensitive to small differences of channel depths, due to the zero-point energy change, particularly at lower energies. This hypothesis was first formulated by the experimental group of Mauersberger ${ }^{2}$ and was also briefly discussed by Marcus. ${ }^{10}$ First accurate variational calculations of this effect were done by Babikov et al. using the lifetime matrix approach, ${ }^{13-15}$ but only for $J=0$. For $J>0$, Grebenshchikov and Schinke ${ }^{16}$ derived the properties of scattering resonances from the time-dependent wave packet simulation. In order to estimate the partition of resonance width onto $\Gamma_{i}^{A}$ and $\Gamma_{i}^{B}$, they employed 1st order perturbation theory, which gave isotope effects in very good agreement with the experiments. Then, Ivanov and Babikov found a rigorous way to split the resonance width onto $\Gamma_{i}^{A}$ and $\Gamma_{i}^{B}$ and applied it to the dimensionally reduced model of ozone,${ }^{44}$ also at $J>0$. Here we, finally, report the results of extensive full-dimensional calculations of this effect in a broad range of rotational excitations.

It should be realized that a purely vibrational zero-point energy change, $\triangle \mathrm{ZPE}$, is directly relevant for $J=0$ only. For rotationally excited cases, it is better to focus on the energy difference between the tops of the centrifugal barriers in two pathways, $A$ and $B$ (see Fig. 10 in Ref. 14), determined here as $\varepsilon_{0 B}^{\sharp}-\varepsilon_{0 A}^{\sharp}=\Delta \mathrm{ZPE}+E_{B}^{\ddagger}-E_{A}^{\ddagger}$. When rotational excitation increases, the centrifugal barrier in the pathway $A$ grows faster than in the pathway $B$, making $E_{B}^{\ddagger}-E_{A}^{\ddagger}$ negative and squeezing the effective $\triangle \mathrm{ZPE}$ range. ${ }^{14}$ Therefore, in Fig. 6, we plot a reduced difference of the pathway-specific decay rate coefficients

$$
\tilde{\Gamma}=\left(\Gamma_{i}^{A}-\Gamma_{i}^{B}\right) /\left(\Gamma_{i}^{A}+\Gamma_{i}^{B}\right)
$$




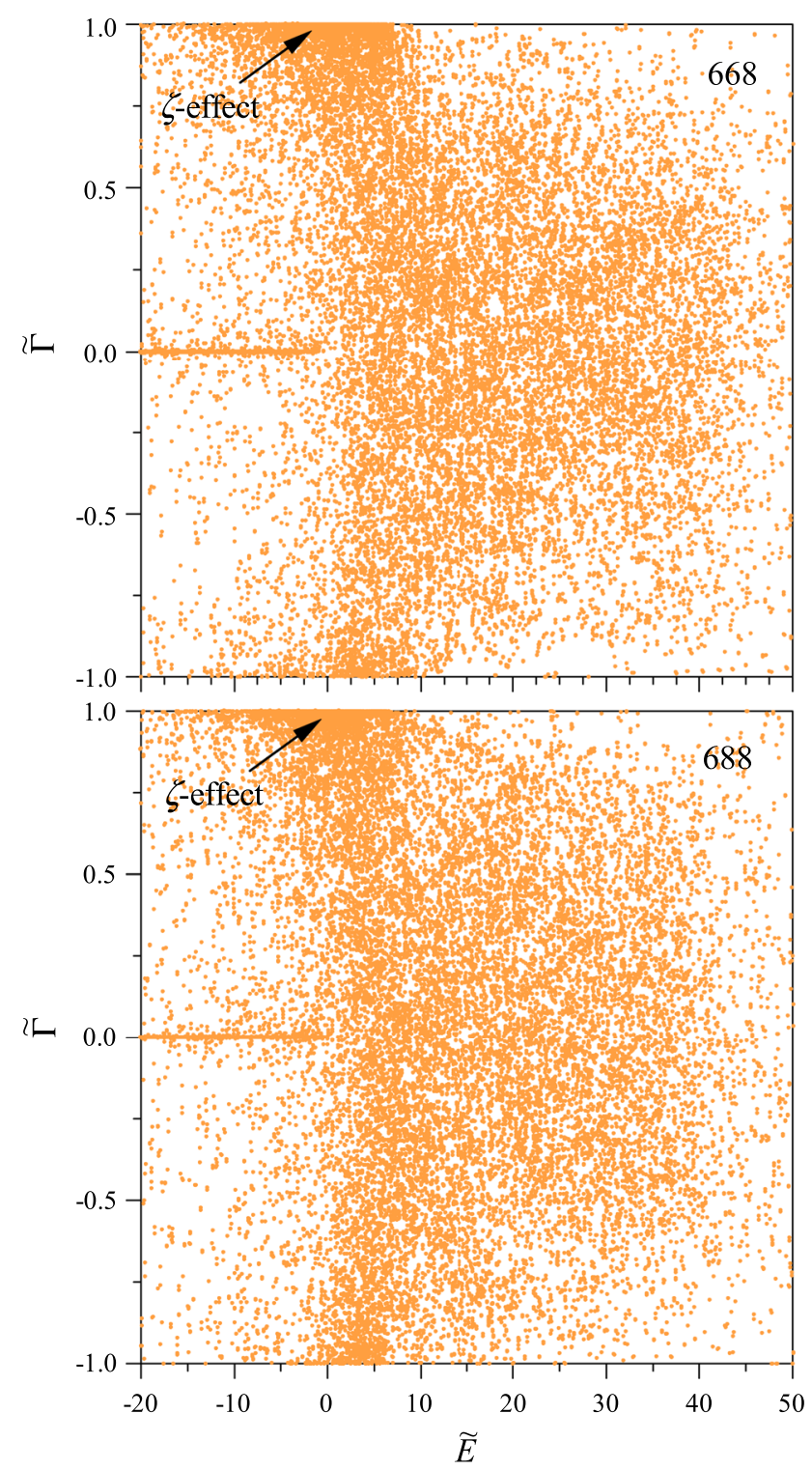

FIG. 6. Demonstration of the fundamental origin of the isotopic $\zeta$-effect. Definitions of $\tilde{\Gamma}$ (reduced difference of the pathway-specific decay rate coefficients) and $\tilde{E}$ (scaled resonance energy) are given in the text. Both are unit-less moieties. Each point of the scatter represents one Feshbach resonance. Properties of all resonances are different, but the asymmetry of the scatter at lower energies is evident, which indicates the tendency of resonances to decay into the deeper channel. At higher resonance energy, this phenomenon disappears. Upper and lower frames correspond to singly and doubly substituted ozone. For clarity, only important states are included, with $P^{\text {well }}>0.05$.

versus the scaled energy of the resonance

$$
\tilde{E}=\left(E_{i}-\varepsilon_{0 A}^{\ddagger}\right) /\left(\varepsilon_{0 B}^{\ddagger}-\varepsilon_{0 A}^{\ddagger}\right) .
$$

Both moieties are unit-less. Note that $\tilde{\Gamma} \rightarrow+1$ corresponds to preferential decay into channel $A$, while $\tilde{\Gamma} \rightarrow-1$ corresponds to preferential decay into channel $B$. The value of $\tilde{\Gamma} \sim 0$ corresponds to nearly equal decay rates and no isotope effect. The values of energy $0 \leq \tilde{E} \leq 1$ correspond to resonances within the rotationally excited analog of the $\triangle \mathrm{ZPE}$ range. Negative $\tilde{E}$ corresponds to tunneling. Large positive values of $\tilde{E}$ correspond to high energy resonances, passing over centrifugal barriers. Figure 6 indicates that different resonances exhibit different properties. Some of them tend to decay more into pathway $A$, and others more into pathway $B$. However, at small energies, mostly in the range $-10 \leq \tilde{E} \leq+5$, we find many more resonances that decay preferentially into the deeper channel, that of pathway $A$. At higher resonance energies $\tilde{E}>+10$, this effect disappears, and the distribution of points along $\tilde{\Gamma}$ becomes almost symmetric. In the lower frame of Fig. 6, which corresponds to doubly substituted ozone, the channels $A$ and $B$ are swapped in the definitions of $\tilde{\Gamma}$ and $\tilde{E}$, to reflect the fact that channel $B$ is deeper in the doubly substituted molecule $\left(K_{\mathrm{ex}}\right.$ $=0.489$ for singly substituted ozone, $K_{\text {ex }}=1.961$ for doubly substituted ozone).

Differences of the pathway-specific formation/decay rate coefficients for asymmetric ozone molecules (resonance widths $\Gamma_{i}^{A}$ and $\Gamma_{i}^{B}$ ) translate into resonance weights of Eq. (14), then into dynamical partition functions $\tilde{Q}_{\mathrm{O}_{3}^{*}}^{A}$ and $\tilde{Q}_{\mathrm{O}_{3}^{*}}^{B}$ of Eqs. (15) and (16), and finally into the rate coefficients $k^{A}$ and $k^{B}$ of Eqs. (10) and (11). At the end, the ratio of $k^{A}$ and $k^{B}$ produces the $\zeta$-effect according to Eq. (1). To gain more insight into this, we also inspected how the value of the ratio $\tilde{Q}_{\mathrm{O}_{3}^{*}}^{A} / \tilde{Q}_{\mathrm{O}_{3}^{*}}^{B}$ for singly substituted ozone, and $\tilde{Q}_{\mathrm{O}_{3}^{*}}^{B} / \tilde{Q}_{\mathrm{O}_{3}^{*}}^{A}$ for doubly substituted ozone, changes as a function of rotational excitation $(J, \Lambda)$. We found that these ratios correlate rather well with the value of the local $\zeta$-effect (i.e., the isotope effect determined for one rotational excitation $J$ and $\Lambda$, rather than the overall $\zeta$-effect summed over the thermal distribution of rotational excitations). We found that these local values of $\zeta(J, \Lambda)$ depend on rotational excitations and vary from almost $\zeta \sim 3$ found near $J=16$ and $\Lambda=16$, down to $\zeta \sim 1$ observed near $J=40$ and $\Lambda=0$. The dependence is not monotonic. The most important contributions to the overall $\zeta$-effect come from moderate rotational excitations around $J \approx 18$ to 20 and $\Lambda \approx 6$ to 8 . When summed over rotational excitations, the resultant isotope effects are $\zeta=1.49$ and $\zeta=1.38$ for singly and doubly substituted isotopomers, respectively, in reasonable agreement with experimental data (see Table I).

In the past, the origin of this effect, named as the $\triangle \mathrm{ZPE}$ effect, has been tentatively attributed to slight exothermicity/endothermicity of the isotope exchange reaction (e.g., ${ }^{16} \mathrm{O}$ $\left.+{ }^{16} \mathrm{O}^{18} \mathrm{O} \leftrightarrow{ }^{16} \mathrm{O}^{16} \mathrm{O}+{ }^{18} \mathrm{O}\right)$ due to the vibrational zeropoint energy change. Here, first of all, we prefer to distance ourselves from this term because a purely vibrational $\triangle \mathrm{ZPE}$ is appropriate for $J=0$ only. Instead, we introduce the $\zeta$-effect that takes rotational excitation into account, by definition in Eq. (1). Next, using the hierarchy of theory levels, we explore several possible sources of the $\zeta$-effect, separately. Using the 1 st theory level, we showed ${ }^{49}$ that slight exothermicity/endothermicity between the pathways $A$ and $B$, by itself, is insufficient to explain the experiment (see Table I). It gave only $\zeta=1.13$ versus $\zeta_{\exp } \sim 1.5$ in the experiment. Now, at the 3rd theory level, we demonstrate that the sensitivity of widths of Feshbach resonances to vibrational and rotational energy differences in two formation pathways $A$ and $B$ provides the remaining and, in fact, major contribution to the $\zeta$-effect. On a qualitative level, our interpretation of this effect is very similar to that of Grebenshchikov and Schinke.

The remaining small discrepancy is, most probably, due to the over-simplified treatment of the stabilization step used 
in this work. Since we are assuming the same value of the stabilization cross section for all resonances, the contributions of higher energy resonances are somewhat overestimated. It would be more reasonable to assume that the scattering resonances at higher energies have smaller values of stabilization cross sections, for example, as determined by single-exponential or double-exponential formulae or other similar models of the ro-vibrational energy transfer. ${ }^{6,18,45}$ Since the $\zeta$-effect is larger for the low energy resonances and disappears at higher energies (see Fig. 6), the employment of a realistic model for energy transfer would lead to some increase of the $\zeta$-effect, in agreement with experiment. In the future, we plan on exploring this issue in more detail.

For the description of the isotopic $\eta$-effect, fundamentally important is the difference of resonance widths for symmetric ozone molecules (decay rate coefficients for pathway $S$, given by $\Gamma_{i}^{S}=1 / 2 \Gamma_{i}$ ) and resonance widths for asymmetric ozone molecules $\Gamma_{i}^{A}$ and $\Gamma_{i}^{B}$ (pathways $A$ and $B$, where $\Gamma_{i}^{A}+\Gamma_{i}^{B}=\Gamma_{i}$ ). These are expected to be somehow sensitive to symmetry $v s$. asymmetry of the formation/decay process (besides the factor of $1 / 2$ introduced at the 0 th level of theory ${ }^{49}$ ). Some hints of this effect have been reported in the past in an approximate study using the dimensionally reduced model of the ozone molecule. ${ }^{44}$ Here we present, for the first time, the results of extensive and accurate calculations that shed more light onto this phenomenon. In Fig. 7, we compare the values of $\Gamma_{i}^{A}, \Gamma_{i}^{B}$, and $\Gamma_{i}^{S}$ for all Feshbach resonances computed for singly and doubly substituted isotopomers of ozone. In order to obtain a meaningful "raster" image of the data, we used the value of $P^{\text {well }}$ as abscissa for this plot, simply because we found that the values of resonance widths correlate well with their probabilities in the well. This picture demonstrates that the values of $\Gamma_{i}^{A}$ and $\Gamma_{i}^{B}$ for asymmetric ozone molecules vary in a rather broad range, which is also consistent with Fig. 6. Indeed, different resonances of asymmetric ozone molecules behave differently. Some of them decay mostly into the pathway $A$ and for those states $\Gamma_{i}^{A} \gg \Gamma_{i}^{B}$ and $\Gamma_{i}^{A} \approx \Gamma_{i}$, and others decay more into pathway $B$ and for those states just opposite is true: $\Gamma_{i}^{A} \ll \Gamma_{i}^{B}$ and $\Gamma_{i}^{B} \approx \Gamma_{i}$. Overall, both large and small values of $\Gamma_{i}^{A}$ and $\Gamma_{i}^{B}$ are found in the distribution. In contrast to this behavior, resonances of symmetric ozone molecules must decay equally into two equivalent decay channels of the pathway $S$ (see the reaction formula in the Introduction). So, we will always find $\Gamma_{i}^{S}=1 / 2 \Gamma_{i}$ for symmetric molecules. Figure 7 reflects this, indicating much narrower distribution of blue points along the vertical axis (pathway $S$ ) compared to the distributions of red and green points (pathways $A$ and $B$ ).

These differences between resonance widths of symmetric and asymmetric molecules, again, translate into the resonance weights of Eq. (14), the dynamical partition functions $\tilde{Q}_{\mathrm{O}_{3}^{*}}^{A}$, $\tilde{Q}_{\mathrm{O}_{3}^{*}}^{B}$, and $\tilde{Q}_{\mathrm{O}_{3}^{*}}^{S}$ of Eqs. (15) and (16), and finally into the rate coefficients $k^{A}, k^{B}$, and $k^{S}$ of Eqs. (10)-(12). At the very end, their ratio produces the $\eta$-effect, according to Eq. (2). We carefully examined how the rotational excitation, determined by $J$ and $\Lambda$, influences the value of the local isotope effect, $\eta(J, \Lambda)$. We found that in the singly substituted ozone, this isotope
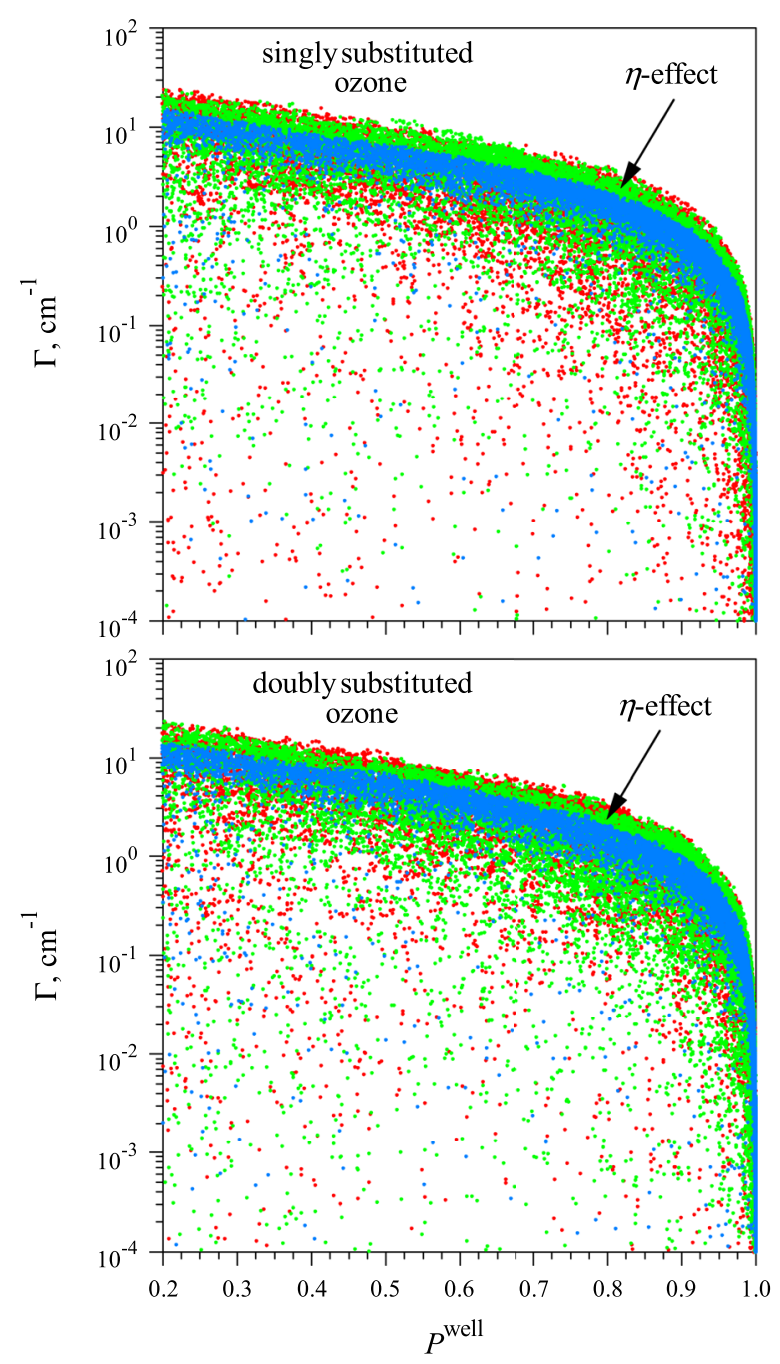

FIG. 7. Demonstration of the possible origin of the isotopic $\eta$-effect. The values of resonance widths $\Gamma_{i}$ (decay rate coefficients) correlate rather well with $P^{\text {well }}$. Each point of the scatter represents one Feshbach resonance. Green and red colors correspond to $\Gamma_{i}^{A}$ and $\Gamma_{i}^{B}$ for an asymmetric ozone isotopologue. The blue color gives $\Gamma_{i} / 2$ for a symmetric ozone isotopologue (rate coefficient of decay per channel, or $\Gamma_{i}^{S}$ ). Upper and lower frames correspond to singly and doubly substituted ozone. One can see that the values of $\Gamma_{i}^{S}$ are represented by a more compact scatter, while $\Gamma_{i}^{A}$ and $\Gamma_{i}^{B}$ exhibit a much broader spread of values.

effect progressively increases from $\eta \sim 0.9$ near $J=16$ and $\Lambda=16$ up to $\eta \sim 1.15$ near $J=40$ and $\Lambda=0$. In doubly substituted ozone, the dependence is almost opposite: the isotope effect progressively decreases from $\eta \sim 1.4$ near $J=16$ and $\Lambda=16$ down to $\eta \sim 1.04$ near $J=40$ and $\Lambda=0$. We found that these changes are rather monotonic but are not identical. When summed over rotational excitations, the resultant isotope effects are $\eta=1.03$ and $\eta=1.12$ for singly and doubly substituted isotopomers, respectively.

These numbers have to be gaged $v s$. results of the 1st theory level, where the effect of mass was taken into account in a statistical rather than dynamical fashion. There, at the 1st theory level, we had $\eta=0.95$ and $\eta=1.04$ for singly and doubly substituted isotopomers, respectively (see Table I). We see that the inclusion of widths of Feshbach resonances leads to very similar increases of $\eta$ values, by +0.080 for both singly and doubly substituted isotopomers. These similar changes make 
sense since resonance widths in Figs. 6 and 7 behave very similar for singly and doubly substituted isotopomers. So their effect is expected to be similar too. Comparison with experimental data in Table I indicates that the effect of resonance width, 3rd level theory, pushes theoretical predictions of the $\eta$-effect in the right direction.

\section{The sensitivity studies}

In this subsection, in order to strengthen our understanding of the influence of resonance properties onto recombination kinetics and on the anomalous isotope effects and in order to roughly estimate the effect of those theory elements that are outside of the scope of this work, we conducted a series of relatively simple but quite insightful computational experiments. In these experiments, we intentionally modified the properties of resonances (computed at the 3rd level of theory, with Feshbach resonances enabled) in order to check the effect of each property or feature, individually. Results are summarized in Table III below and can be compared to those in Table I.

Experiment (i): Instead of using the accurately computed values of $\Gamma_{i}^{A}$ and $\Gamma_{i}^{B}$ for resonances in asymmetric ozone molecules, we set them equal to each other and to one half of the total resonance width: $\Gamma_{i}^{A}=\Gamma_{i}^{B}=\frac{1}{2} \Gamma_{i}$. With this ansatz, we recomputed the values of recombination rate coefficients and determined the resultant $\zeta$-effect and $\eta$-effect. Results are presented in the first row of Table III. We see that the values of the $\eta$-effect remained basically unaffected by this modification (decreased by $1 \%$ only). However, the large $\zeta$-effect disappeared almost completely (only 3\% remains, out of almost $50 \%$ ). This demonstrates very clearly that the $\zeta$-effect is driven mostly by an unequal splitting of resonance width $\Gamma_{i}$ for asymmetric ozone molecules onto the pathway-specific widths $\Gamma_{i}^{A}$ and $\Gamma_{i}^{B}$.

Experiment (ii): Instead of computing the weights of resonances accurately, using Eq. (13), we set $w_{i}=1$ for all resonances of symmetric ozone molecules (pathway $S$ ). For the resonances of asymmetric ozone molecules (participating in the pathways $A$ and $B$ ), the total weight is also set to $w_{i}=1$, but it is split between the pathways $A$ and $B$ proportionally to the values of $\Gamma_{i}^{A}$ and $\Gamma_{i}^{B}$. Results obtained with this assumption are presented in the second row of Table III. We see that the values of the $\eta$-effect, again, did not change much (decreased by few percent). However, the $\zeta$-effect has substantially increased, reaching the range of experimental values. Technically, this modification was achieved by setting $[\mathrm{M}]=0$ in Eq. (13), but

TABLE III. Two isotope effects in singly and doubly substituted ozone molecules, computed at the 3rd level of theory with several minor modifications, as outlined in the description of three computational experiments in the text.

\begin{tabular}{|c|c|c|c|c|c|}
\hline \multicolumn{2}{|r|}{ Computational experiment } & \multicolumn{2}{|c|}{$\zeta$-Effect } & \multicolumn{2}{|c|}{$\eta$-Effect } \\
\hline No & Model/meaning & Single & Double & Single & Double \\
\hline $\mathrm{i}$ & $\Gamma_{i}^{A}=\Gamma_{i}^{B}=\frac{1}{2} \Gamma_{i}$ & 1.03 & 1.02 & 1.02 & 1.11 \\
\hline ii & $w_{i}=1$, or $[\mathrm{M}]=0$ & 1.72 & 1.55 & 1.00 & 1.08 \\
\hline iii & Van der Waals states (estimate) & 1.43 & 1.29 & 0.97 & 1.12 \\
\hline iv & Geometric phase (estimate) & 1.62 & 1.27 & 0.99 & 1.06 \\
\hline $\mathrm{v}$ & Channel interference (estimate) & 1.55 & 1.31 & 0.95 & 0.97 \\
\hline
\end{tabular}

the goal was not to explore the low-pressure limit per se. Our purpose in this experiment was to make narrower resonances as important for the recombination process as broader resonances are. (Extremely narrow resonances with $\Gamma_{i}<10^{-5} \mathrm{~cm}^{-1}$, that make negligible contributions to the recombination process under normal conditions, were completely removed from consideration). Indeed, narrower resonances are found at lower energies where the $\zeta$-effect is more significant (see Fig. 6), but their weights are smaller due to small values of $\Gamma_{i}$. So, the artificial lift of their weights leads to the increase of the $\zeta$-effect.

Experiment (iii): Instead of neglecting the delocalized van der Waals states completely, here we try to take into account the contributions of some of them. Namely, for lower rotational excitations, when the top of the centrifugal barrier is below dissociation threshold, we will assume that the van der Waals states can be quenched into the stable ozone molecules in the main (covalent) well as efficiently as the localized resonances. For higher rotational excitations, with the centrifugal barrier sticking above the asymptote, we will still say that the stabilization of van der Waals states is inefficient. This modification increases the absolute values of recombination rate coefficients substantially (doubles them, roughly). The resultant isotope effects are presented in the third row of Table III. Comparison with results of the 3rd theory level (Table I) indicates that the inclusion of the van der Waals States is very unlikely to improve comparison with experiment. The values of the $\zeta$ effect and $\eta$-effect are both slightly reduced, but differently for the singly and doubly substituted ozone molecules.

Experiment (iv): In this test, we are going to estimate a possible effect of the geometric phase on the pathway $B$ by removing the contributions of symmetric vibrational states [A $\mathrm{A}_{1}$, indicated by solid curves in Fig. 1(b)] and doubling the contributions of antisymmetric vibrational states $\left[\mathrm{B}_{1}\right.$, indicated by dashed curves in Fig. 1(b)]. The reason for doing this is that solid curves in Fig. 1(b) correlate asymptotically with even rotational states of homo-nuclear $\mathrm{O}_{2}$ that are forbidden by symmetry. ${ }^{13,26}$ Only pathway $B$ is affected by this modification. There is no simple way of taking this effect into account in the electronically adiabatic single-surface calculations $^{23,46}$ because the electronic state symmetry of the PES changes from being symmetric over the covalent well to being antisymmetric in the dissociation asymptote. What we propose here is just an estimate, based on the data we have at hand. Results are given in the fourth row of Table III. Relative to the unaltered results of the 3rd theory level (see Table I), the $\zeta$-effect changes in the opposite directions for singly and doubly substituted molecules: increases by $13 \%$ and decreases by $11 \%$, respectively. This appreciable change is understood since the vibrational symmetry $\mathrm{A}_{1}$ (removed in this experiment) is the only contributor in the cases of rotations with $\Lambda=0$ (any $J$ values). This reduces the value of $k^{B}$, which in turn increases the $\zeta$-effect in the case of singly substituted ozone where the channel of pathway $B$ is shallower, but at the same time, decreases the $\zeta$-effect in the case of doubly substituted ozone where the channel of pathway $B$ is deeper. By contrast, the $\eta$-effect is decreased in both cases, by close to $5 \%$. Thus, no source of the large isotopic $\eta$-effect is likely to be related to the geometric phase. 
Experiment (v): Here, for the pathways $A$ and $S$ only, we will neglect the contributions of antisymmetric vibrational states $\left(\mathrm{B}_{1}\right)$ and simultaneously double the contributions of symmetric vibrational states $\left(\mathrm{A}_{1}\right)$, in order to determine the effect of vibrational symmetry. The reason for trying this is that the pathways $A$ and $S$ connect towards two physically equivalent (indistinguishable) formation/decay channels and the corresponding fluxes interfere in the interaction region. This interference occurs differently in the cases of symmetric and anti-symmetric vibrational states. It was argued in the past ${ }^{47}$ that such interferences may be a source of the $\eta$-effect. Results are presented in the last row of Table III. We see that the $\zeta$-effect changes in opposite directions for singly and doubly substituted ozone molecules, similar to the previous experiment and, basically, for the same reason. Interestingly, the $\eta$-effect is decreased quite substantially in both molecules, opposite to experimental data.

\section{CONCLUSIONS}

In this paper, as expansion of simpler statistical theory developed earlier and reported elsewhere, ${ }^{49}$ we developed and implemented two advanced levels of theory for the description of the anomalously large isotope effects known to occur in the recombination reaction that forms ozone. These isotope effects (most notably the $\zeta$-effect and $\eta$-effect) are responsible for the mass-independent fractionation of oxygen isotopes in the atmosphere of Earth, which has a significant applied importance in atmospheric chemistry, geochemistry, and astrochemistry. The fundamental understanding of the molecular level origin of these effects is a long-standing problem in physical chemistry, too. The two levels of theory developed here help to understand the role of tunneling, or "shape" resonances and, separately the role of vibrational channel couplings, or Feshbach resonances in the ozone molecule.

Pure shape resonances (with vibrational couplings disabled) are found to contribute little to the overall recombination process since they have to be populated by tunneling and occur rather unfrequently in the spectrum, in the vicinity of the top of centrifugal barrier only. The resultant isotope effects are found to be somewhat accidental and, importantly, they do not look like experimental data for singly and doubly substituted ozone molecules. In particular, at this level of theory, the value of the $\eta$-effect is 0.73 for both singly and doubly substituted molecules, which is in the wrong direction compared to the experiment, where the value of $\eta_{\exp }=1.16$ is observed. This source of anomalous isotope effect can be ruled out.

When the vibrational couplings are included and the mechanism of Feshbach resonances is enabled, the rate coefficients of the process increase dramatically. Feshbach-type resonances are found to occur in a much broader range of spectrum, and their density of states is much higher. Properties of Feshbach resonances relevant to the isotopic $\zeta$-effect and $\eta$-effect are studied in detail. At this level of theory, the isotopic $\zeta$-effect is reproduced rather well: $\zeta=1.49$ and 1.38 for singly and doubly substituted molecules, respectively, which is reasonably close to the experimental values of $\zeta_{\exp }=1.55$ and 1.63. Further computational experiments showed that if the weights of narrower resonances are increased, then $\zeta$-effects can reach 1.72 and 1.55 , which is in the range of experimental data. Incorporation of the more sophisticated model for ro-vibrational energy transfer (instead of strong collision assumption employed here) may have a similar effect on $\zeta$ values. This question will be explored in the near future but, overall, $\zeta$-effects seem to be well understood at this point. Its origin is in unequal splitting of resonance formation/decay rates, determined by resonance widths, for two competing reaction pathways $A$ and $B$.

The isotopic $\eta$-effect is a harder puzzle. A statistical description of the process presented elsewhere ${ }^{49}$ indicates $\eta=$ 0.95 and 1.04 that deviate from the value of 1.00 in opposite directions for singly and doubly substituted ozone molecules, in contrast to the experimental data where $\eta_{\exp }=1.16$ was observed for both molecules. In this paper, the inclusion of Feshbach resonances increases the values of $\eta$ for both singly and doubly substituted molecules rather consistently, by +0.08 , which pushes theoretical prediction in the right direction. This finding is explained by a slightly different partition of the decay rates of the resonances in symmetric and asymmetric ozone molecules. In symmetric ozone molecules, all resonances decay equally into two equivalent dissociation channels of the pathway $S$, while in asymmetric ozone molecules, some resonances decay more efficiently into the pathway $A$ and very little into the pathway $B$, while other resonances do just the opposite.

This phenomenon, however, is not large enough to match experimental observations. The values predicted by our best theory level at this point are $\eta=1.03$ and 1.12 for singly and doubly substituted ozone molecules. This indicates that there should be some other additional source of the isotopic $\eta$-effect, either in the ozone molecule itself or in the process of resonance stabilization by bath gas collisions. Moreover, our data indicate that this (still mysterious) source should favor the singly substituted lighter ozone molecules over the doubly substituted heavier ozone molecules, in order to compensate for the difference observed between them at the current level of theory. Additional computational experiments reported in Table III showed that our predicted $\eta$-effect is rather stable, insensitive to any "knobs" we have tried to turn so far. Thus, the search for the additional source of the isotopic $\eta$-effect will continue.

One of the possible improvements of the present theory would be to include the Coriolis coupling term, together with the asymmetric-top rotor term (both neglected here), in the kinetic energy operator. These rotation-vibration couplings are responsible for the superposition of the vibrational states with different values of $\Lambda$, within each $J$ value. Such development could be viewed as the next theory level, but one should be aware of a dramatic increase of the computational cost. The resultant Hamiltonian matrix is expected to be larger, by a factor of roughly $\times 10$, with associated numerical diagonalization effort increasing by a factor of roughly $\times 100$. Such calculations are not affordable at this point.

It should also be noted that some discrepancies of the theoretical treatment proposed here with experimental results may be due to the assumption of the Lindemann mechanism for the recombination reaction. There are indications that the 
so-called "chaperon" mechanism may also be responsible for at least a part of the ozone formation rate. ${ }^{20,39,48}$ The quantum mechanical description of scattering resonances in the chaperon mechanism, formed by the weakly bound complexes such as $\mathrm{OM}^{*}$ and $\mathrm{O}_{2} \mathrm{M}^{*}$, where $\mathrm{M}$ is an atom or molecule of the bath gas $\left(\mathrm{N}_{2}\right.$, Ar, etc. $)$, should also be done at some point for a truly complete picture of the process.

\section{SUPPLEMENTARY MATERIAL}

See supplementary material for the computed dependencies of dynamical partition functions $\tilde{Q}^{A}, \tilde{Q}^{B}$, and $\tilde{Q}^{S}$ and recombination rate coefficients $k^{A}, k^{B}$, and $k^{S}$, on rotational excitation $J$ and $\Lambda$, computed using 2 nd and 3rd theory levels for singly and doubly substituted ozone molecules.

\section{ACKNOWLEDGMENTS}

This research was supported by the NSF Atmospheric Chemistry Program, Division of Atmospheric Sciences, Grant No. AGS-1252486. This research used resources of the National Energy Research Scientific Computing Center, which is supported by the Office of Science of the U.S. Department of Energy under Contract No. DE-AC02-05CH11231.

${ }^{1}$ A. Teplukhin and D. Babikov, "Properties of Feshbach and "shape"resonances in ozone and their role in recombination reactions and anomalous isotope effects," Faraday Discuss. (to be published).

${ }^{2}$ C. Janssen, J. Guenther, K. Mauersberger, and D. Krankowsky, Phys. Chem. Chem. Phys. 3, 4718 (2001); see also: B. Tuzson, "Symmetry specific study of ozone isotopomer formation," Ph.D. thesis, University of Heidelberg, 2005.

${ }^{3}$ R. Schinke, S. Y. Grebenshchikov, M. V. Ivanov, and P. Fleurat-Lessard, Annu. Rev. Phys. Chem. 57, 625 (2006).

${ }^{4}$ A. Gross and G. D. Billing, Chem. Phys. 217, 1 (1997).

${ }^{5}$ T. A. Baker and G. I. Gellene, J. Chem. Phys. 117, 7603 (2002).

${ }^{6}$ M. V. Ivanov, S. Y. Grebenshchikov, and R. Schinke, J. Chem. Phys. 120, 10015 (2004).

${ }^{7}$ M. Ivanov and R. Schinke, J. Chem. Phys. 122, 234318 (2005).

${ }^{8}$ M. Kryvohuz and R. Marcus, J. Chem. Phys. 132, 224305 (2010).

${ }^{9}$ B. C. Hathorn and R. A. Marcus, J. Chem. Phys. 111, 4087 (1999).

${ }^{10}$ B. C. Hathorn and R. A. Marcus, J. Chem. Phys. 113, 9497 (2000).

${ }^{11}$ Y. Q. Gao and R. A. Marcus, Science 293, 259 (2001).

${ }^{12}$ Y. Q. Gao and R. A. Marcus, J. Chem. Phys. 116, 137 (2002).

${ }^{13}$ D. Babikov, B. K. Kendrick, R. B. Walker, R. T. Pack, P. Fleurat-Lesard, and R. Schinke, J. Chem. Phys. 118, 6298 (2003).

${ }^{14}$ D. Babikov, B. K. Kendrick, R. B. Walker, R. T. Pack, P. Fleurat-Lesard, and R. Schinke, J. Chem. Phys. 119, 2577 (2003).

${ }^{15}$ D. Babikov, B. K. Kendrick, R. B. Walker, R. Schinke, and R. T Pack, Chem. Phys. Lett. 372, 686 (2003).
${ }^{16}$ S. Y. Grebenshchikov and R. Schinke, J. Chem. Phys. 131, 181103 (2009).

${ }^{17}$ M. V. Ivanov and R. Schinke, Mol. Phys. 108, 259 (2010).

${ }^{18}$ M. V. Ivanov and D. Babikov, J. Chem. Phys. 144, 154301 (2016).

${ }^{19}$ M. Tashiro and R. Schinke, J. Chem. Phys. 119, 10186 (2003).

${ }^{20}$ M. V. Ivanov and R. Schinke, J. Chem. Phys. 124, 104303 (2006).

${ }^{21}$ R. T. Pack, R. B. Walker, and B. K. Kendrick, J. Chem. Phys. 109, 6714 (1998).

${ }^{22}$ V. J. Barclay, C. E. Dateo, I. P. Hamilton, B. Kendrick, R. T Pack, and D. W. Schwenke, J. Chem. Phys. 103, 3864 (1995).

${ }^{23}$ B. K. Kendrick, J. Chem. Phys. 112, 5679 (2000).

${ }^{24}$ A. Teplukhin and D. Babikov, J. Chem. Phys. 145, 114106 (2016).

${ }^{25}$ S. Ndengué, R. Dawes, X.-G. Wang, T. Carrington, Z. Sun, and H. Guo, J. Chem. Phys. 144, 074302 (2016).

${ }^{26}$ D. Lapierre, A. Alijah, R. Kochanov, V. Kokoouline, and V. Tyuterev, Phys. Rev. A 94, 042514 (2016).

${ }^{27}$ D. Charlo and D. C. Clary, J. Chem. Phys. 117, 1660 (2002).

${ }^{28}$ D. Charlo and D. C. Clary, J. Chem. Phys. 120, 2700 (2004).

${ }^{29}$ T. Xie and J. M. Bowman, Chem. Phys. Lett. 412, 131 (2005).

${ }^{30}$ M. Ivanov, S. Y. Grebenshchikov, and R. Schinke, J. Chem. Phys. 130, 174311 (2009).

${ }^{31}$ R. Schinke and P. Fleurat-Lessard, J. Chem. Phys. 122, 094317 (2005).

${ }^{32}$ M. V. Ivanov and D. Babikov, J. Chem. Phys. 136, 184304 (2012).

${ }^{33}$ T. Chu and K. Han, Annu. Rep. Prog. Chem., Sect. C: Phys. Chem. 108, 10 (2012).

${ }^{34}$ B. K. Kendrick, J. Hazra, and N. Balakrishnan, Nat. Commun. 6, 7918 (2015).

${ }^{35}$ J. C. Juanes-Marcos, S. C. Althorpe, and E. Wrede, Science 309, 1227 (2005).

${ }^{36}$ A. Kuppermann, in Potential Energy Surfaces and Dynamics Calculations, edited by D. G. Truhlar (Plenum Press, New York, 1981), p. 375.

${ }^{37}$ Resonances in Electron-Molecule Scattering, van der Waals Complexes, and Reactive Chemical Dynamics, edited by D. G. Truhlar (American Chemical Society, Washington, 1984).

${ }^{38}$ E. Vetoshkin and D. Babikov, Phys. Rev. Lett. 99, 138301 (2007).

${ }^{39}$ A. Teplukhin and D. Babikov, Phys. Chem. Chem. Phys. 18, 19194 (2016).

${ }^{40}$ Z. Bačić and J. C. Light, J. Chem. Phys. 87, 4008 (1987), and references there in.

${ }^{41}$ J. C. Light and T. Carrington, in Advances in Chemical Physics, edited by I. Prigogine and S. A. Rice (John Wiley \& Sons, Inc., Hoboken, NJ, USA, 2000), pp. 263-310.

${ }^{42}$ V. Kokouline and C. H. Green, Phys. Rev. A 68, 012703 (2003).

${ }^{43}$ M. V. Ivanov and D. Babikov, J. Chem. Phys. 134, 174308 (2011).

${ }^{44}$ M. V. Ivanov and D. Babikov, Proc. Natl. Acad. Sci. U. S. A. 110, 17708 (2013).

${ }^{45}$ Z. Zhu and R. A. Marcus, J. Chem. Phys. 129, 214106 (2008).

${ }^{46}$ D. Babikov, B. Kendrick, P. Zhang, and K. Morokuma, J. Chem. Phys. 122, 044315 (2005).

${ }^{47}$ P. Reinhardt and F. Robert, Earth Planet. Sci. Lett. 368, 195 (2013).

${ }^{48}$ K. Luther, K. Oum, and J. Troe, Phys. Chem. Chem. Phys. 7, 2764-2770 (2005).

${ }^{49}$ A. Teplukhin and D. Babikov, "Several levels of theory for description of isotope effects in ozone: Symmetry effect and Mass effect," J. Phys. Chem. A (to be published). 